\title{
Extension of Analytical Target Cascading using Augmented Lagrangian Coordination for Multidisciplinary Design Optimization
}

\author{
S. Tosserams ${ }^{1}$, M. Mokkolaras ${ }^{2 \dagger} \nmid$ L.F.P. Etman ${ }^{1} ;$ J.E. Rooda ${ }^{1 \S}$ \\ ${ }^{1}$ Department of Mechanical Engineering, Eindhoven University of Technology, The Netherlands \\ 2 Department of Mechanical Engineering, The University of Michigan, Ann Arbor, MI
}

\begin{abstract}
Analytical target cascading (ATC) is a method originally developed for translating system-level design targets to design specifications for the elements comprising the system. ATC has also been shown to be useful for coordinating distributed design optimization of hierarchical, multilevel systems. The traditional ATC formulation uses a hierarchically decomposed problem structure, in which coordination is performed by communicating target and response values between parents and children. This paper presents two extensions of the ATC formulation to allow non-hierarchical target-response coupling between subproblems and to introduce system-wide constraints that depend on local variables of two or more subproblems. The ATC formulation with these extensions belongs to a subclass of augmented Lagrangian coordination, and has thus converge properties under the usual convexity and continuity assumptions. A supersonic business jet design problem reported earlier in the literature is used to illustrate these extensions.
\end{abstract}

\section{Introduction}

The development process of engineering systems often follows a decomposition paradigm. The elements that comprise the system are developed individually, and then integrated to form the system. Accordingly, the optimal system design problem is decomposed into smaller subproblems, each associated with a the design of a part of the system. These design subproblems are then solved autonomously, and a systematic coordination is required to guide the subproblems towards a design that is consistent and optimal for the system as a whole.

Analytical target cascading (ATC) is a method originally developed for translating system-level design targets to design specifications for the elements that comprise the system. ${ }^{1-4}$ ATC has also been shown to be useful as a coordination method for distributed design optimization of hierarchical, multilevel systems. ${ }^{5,6}$ In the ATC paradigm, design targets are cascaded using a multilevel hierarchical decomposition. Subproblems associated with the elements of the system not only determine targets for their children, but also compute responses to targets they receive from their parents. These responses are rebalanced up, and the objective of a subproblem is to minimize the deviations between the target-response pairs while maintaining feasibility with respect to its local design constraints. The process of exchanging targets and responses between subproblems can be shown to converge to optimal system solutions with arbitrarily small deviations between targets and responses under the usual convexity and continuity assumptions. ${ }^{3,5,7}$

The traditional ATC formulation uses a hierarchically decomposed problem structure, in which coordination is performed by communicating target and response values between parents and children. "Hierarchical" refers to the functional dependency among system elements: responses of elements higher in the hierarchy depend on responses of elements lower in the hierarchy, but not vice versa (see Figure 1(a)). To increase the flexibility of the ATC formulation beyond this hierarchical structure, two formulation extensions are presented in this paper: non-hierarchical targetresponse coupling and system-wide functions.

The traditional ATC process may not be the most suitable way to coordinate problems that do not have a clear hierarchical structure. For those problems, direct communication between subproblems may be more appropriate. For example, typical multidisciplinary design optimization (MDO) problems are composed of subproblems ordered

${ }^{*}$ PhD student, Department of Mechanical Engineering, Eindhoven University of Technology, E-mail: s.tosserams@tue.nl

${ }^{\dagger}$ Associate Research Scientist, Department of Mechanical Engineering, University of Michigan, E-mail: mk@umich.edu

¥Assistant Professor, Department of Mechanical Engineering, Eindhoven University of Technology, E-mail: 1.f.p.etman@tue.nl

${ }^{\S}$ Professor, Department of Mechanical Engineering, Eindhoven University of Technology, E-mail: j.e.rooda@tue.nl 


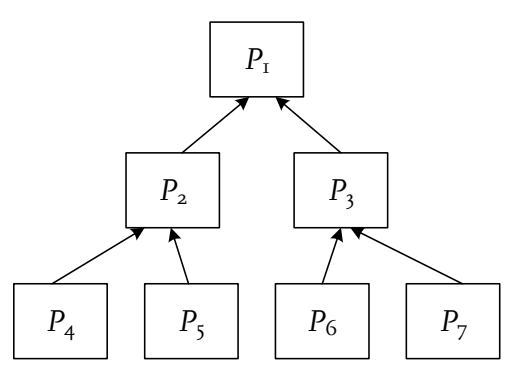

(a) Hierarchical

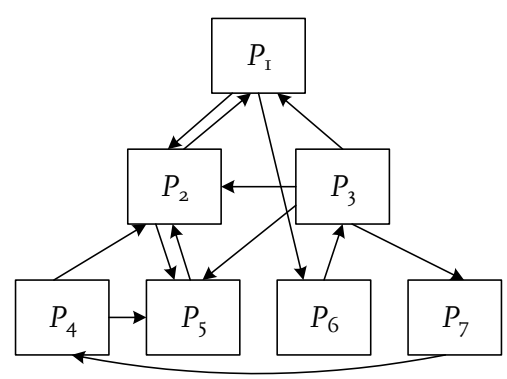

(b) Non-hierarchical

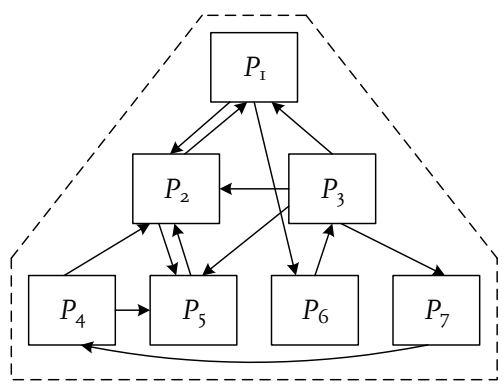

(c) Non-hierarchical with system-wide functions

Figure 1: Illustration of the structure of the original ATC formulation, and the proposed extensions. Arrows indicate the flow of subproblem responses, and the dashed box in the rightmost figure is used to represent the existence of system-wide functions.

by analysis disciplines between which no clear hierarchy may exist. The first goal of this paper is therefore to extend the ATC formulation to include non-hierarchical target-response coupling between subproblems such that nonhierarchical functional dependencies between subproblems are possible (see Figure 1(b)).

The second formulation extension considers the interaction between subproblems through system-wide functions. While ATC coordinates coupling though target and response variables, some problems may be coupled through a set of system-wide functions that may depend on the local variables of more than one subproblem. Such coupling functions often depend on a system performance measure such as mass, cost, volume, or power. Coordinating system-wide functions through target variables in the hierarchical structure may require the introduction of many copies of local variables, which can increase the size of the individual subproblems. This paper presents a more direct approach for the coordination of system-wide functions that does not require the introduction of variable copies (see Figure 1(c)).

Each of the above extended ATC formulations is demonstrated to belong to a subclass of the augmented Lagrangian coordination $\left(\mathrm{ALC}^{8}\right)$ formulation. ALC algorithms and convergence theory apply directly to the extended ATC formulation, which can therefore be guaranteed to converge. The extensions and their motivation are illustrated using the design of a supersonic business jet problem of Ref. 9.

\section{Formulation extensions for analytical target cascading}

We begin with the ATC subproblem in its augmented Lagrangian formulation of Ref. 5:

$$
\begin{aligned}
\min _{\overline{\mathbf{x}}_{i j}} & f_{i j}\left(\overline{\mathbf{x}}_{i j}\right)+\phi\left(\mathbf{t}_{i j}-\mathbf{r}_{i j}\right)+\sum_{k \in \mathscr{C}_{i j}} \phi\left(\mathbf{t}_{(i+1) k}-\mathbf{r}_{(i+1) k}\right) \\
\text { subject to } & \mathbf{g}_{i j}\left(\overline{\mathbf{x}}_{i j}\right) \leq \mathbf{0} \\
& \mathbf{h}_{i j}\left(\overline{\mathbf{x}}_{i j}\right)=\mathbf{0} \\
& \mathbf{r}_{i j}=\mathbf{a}_{i j}\left(\overline{\mathbf{x}}_{i j}\right) \\
\text { where } & \overline{\mathbf{x}}_{i j}=\left[\mathbf{x}_{i j}, \mathbf{r}_{i j}, \mathbf{t}_{(i+1) k_{1}}, \ldots, \mathbf{t}_{\left.(i+1) k_{c_{i j}}\right]}\right.
\end{aligned}
$$

where $\overline{\mathbf{x}}_{i j}$ are the optimization variables for subproblem $j$ at level $i, \mathbf{x}_{i j}$ are local design variables, $\mathbf{r}_{i j}$ are response variables related to the targets $\mathbf{t}_{i j}$ computed by the parent of subproblem $j$. Subproblem $j$ computes targets $\mathbf{t}_{(i+1) k}$ for its children $k \in \mathscr{C}_{i j}$ at level $i+1$ that in turn compute responses $\mathbf{r}_{(i+1) k}$. Function $f_{i j}$ is the local objective for subproblem $j$, and $\mathbf{g}_{i j}$ and $\mathbf{h}_{i j}$ are local inequality and equality constraints. Functions $\mathbf{a}_{i j}$ are analysis models used to compute the responses $\mathbf{r}_{i j}$ of subproblem $j$ for its parent. $\phi\left(\mathbf{t}_{i j}-\mathbf{r}_{i j}\right)=\mathbf{v}_{i j}^{T}\left(\mathbf{t}_{i j}-\mathbf{r}_{i j}\right)+\left\|\mathbf{w}_{i j} \circ\left(\mathbf{t}_{i j}-\mathbf{r}_{i j}\right)\right\|_{2}^{2}$ is an augmented Lagrangian function on the inconsistencies $\mathbf{c}_{i j}=\mathbf{t}_{i j}-\mathbf{r}_{i j}$, where $\mathbf{v}_{i j}$ and $\mathbf{w}_{i j}$ are penalty parameters.

For brevity of notation, the level index $i$ is dropped in the following sections. This does not introduce ambiguity since the use of the subproblem index $j$ suffices to uniquely identify each subproblem.

\section{A. Non-hierarchical subproblem coupling}

Although ATC has been primarily developed for product design problems with a hierarchical structure, extensions to non-hierarchical problems are possible. We extend ATC subproblems such that they can send and receive targets from 
any other subproblem, instead of just their parent or children. In such a case, subproblems have neighbors between which targets and responses are communicated. Since any subproblem can send targets or responses to any other subproblem, we use a double index notation for target and response variables to denote the direction of communication. The first index denotes the sending subproblem and the second index denotes the receiving subproblem: $\mathbf{t}_{j n}$ are the targets sent from subproblem $j$ to subproblem $n$, and $\mathbf{r}_{n j}$ are the responses computed by subproblem $n$ to match the aforementioned targets. Note that the traditional ATC formulation of (1) does not require such a notation since targets are always set by parents, and responses are always computed by children.

Furthermore, let $\mathscr{N}_{j}^{\mathrm{t}}$ be the set of neighbors for which subproblem $j$ sets targets, and let $\mathscr{N}_{j}^{\mathrm{r}}=\bigcup_{n=1}^{M}\left\{j \mid j \in \mathscr{N}_{n}^{\mathrm{t}}\right\}$ be the set of neighbors from which subproblem $j$ receives targets. The ATC subproblem for non-hierarchical subproblem coupling then becomes

$$
\begin{aligned}
\min _{\overline{\mathbf{x}}_{j}} & f_{j}\left(\overline{\mathbf{x}}_{j}\right)+\sum_{n \in \mathscr{N}_{j}^{\mathrm{r}}} \phi\left(\mathbf{t}_{n j}-\mathbf{r}_{j n}\right)+\sum_{n \in \mathscr{N}_{j}^{\mathrm{t}}} \phi\left(\mathbf{t}_{j n}-\mathbf{r}_{n j}\right) \\
\text { subject to } & \mathbf{g}_{j}\left(\overline{\mathbf{x}}_{j}\right) \leq \mathbf{0} \\
& \mathbf{h}_{j}\left(\overline{\mathbf{x}}_{j}\right)=\mathbf{0} \\
& \mathbf{r}_{j n}=\mathbf{S}_{j}^{n} \mathbf{a}_{j}\left(\overline{\mathbf{x}}_{j}\right) \quad n \in \mathscr{N}_{j}^{\mathrm{r}} \\
& \overline{\mathbf{x}}_{j}=\left[\mathbf{x}_{j}, \mathbf{r}_{j n}\left|n \in \mathscr{N}_{j}^{\mathrm{r}}, \mathbf{t}_{j n}\right| n \in \mathscr{N}_{j}^{\mathrm{t}}\right]
\end{aligned}
$$

where $\mathbf{S}_{j}^{n}$ is a binary selection matrix that selects components from $\mathbf{a}_{j}$ that are sent to subproblem $n$. Common subproblems in sets $\mathscr{N}_{j}^{\mathrm{t}}$ and $\mathscr{N}_{j}^{\mathrm{r}}$ indicate feedback coupling between subproblem $j$ and the common subproblem. Observe that if $\mathscr{N}_{j}^{\mathrm{t}}=\mathscr{C}_{i j}$ and $\mathscr{N}_{j}^{\mathrm{r}}=\left\{p_{j}\right\}$, where $p_{j}$ denotes the parent of $j$, then the subproblem reduces to the original ATC subproblem formulation of (1). Furthermore, special cases of the above formulation are the ATC formulation for product family design presented in Ref. 10, and the ATC formulation of Ref. 6 that allows feedback targets between parents and children.

\section{B. System-wide functions}

System-wide functions are objectives or constraints that depend on the variables of more than one subproblem. In the extended ATC formulation proposed here, system-wide objectives $f_{0}\left(\overline{\mathbf{x}}_{1}, \ldots, \overline{\mathbf{x}}_{M}\right)$ can be included directly in the objective of a subproblem. System-wide constraints $\mathbf{g}_{0}\left(\overline{\mathbf{x}}_{1}, \ldots, \overline{\mathbf{x}}_{M}\right)$ and $\mathbf{h}_{0}\left(\overline{\mathbf{x}}_{1}, \ldots, \overline{\mathbf{x}}_{M}\right)$ are relaxed with an augmented Lagrangian penalty function, which is included in the subproblem objectives as well. The ATC subproblem formulation that allows system-wide functions is given by

$$
\begin{aligned}
\min _{\overline{\mathbf{x}}_{j}} & f_{j}\left(\overline{\mathbf{x}}_{j}\right)+\sum_{n \in \mathscr{N}_{j}^{\mathrm{r}}} \phi\left(\mathbf{t}_{n j}-\mathbf{r}_{j n}\right)+\sum_{n \in \mathscr{N}_{j}^{\mathrm{t}}} \phi\left(\mathbf{t}_{j n}-\mathbf{r}_{n j}\right) \\
& +f_{0}\left(\overline{\mathbf{x}}_{1}, \ldots, \overline{\mathbf{x}}_{M}\right)+\phi\left(\mathbf{g}_{0}\left(\overline{\mathbf{x}}_{1}, \ldots, \overline{\mathbf{x}}_{M}\right)+\mathbf{s}_{0}^{2}\right)+\phi\left(\mathbf{h}_{0}\left(\overline{\mathbf{x}}_{1}, \ldots, \overline{\mathbf{x}}_{M}\right)\right) \\
\text { subject to } & \mathbf{g}_{j}\left(\overline{\mathbf{x}}_{j}\right) \leq \mathbf{0} \\
& \mathbf{h}_{j}\left(\overline{\mathbf{x}}_{j}\right)=\mathbf{0} \\
& \mathbf{r}_{j n}=\mathbf{S}_{j}^{n} \mathbf{a}_{j}\left(\overline{\mathbf{x}}_{j}\right) \quad n \in \mathscr{N}_{j}^{\mathrm{r}} \\
& \overline{\mathbf{x}}_{j}=\left[\mathbf{x}_{j}, \mathbf{r}_{j n}\left|n \in \mathscr{N}_{j}^{\mathrm{r}}, \mathbf{t}_{j n}\right| n \in \mathscr{N}_{j}^{\mathrm{t}}\right]
\end{aligned}
$$

where the slack variables $\mathbf{s}_{0}$ are used to allow negative (feasible) values for the system-wide inequality constraints $\mathbf{g}_{0}$. The vector $\mathbf{s}_{j}$ includes the slack variables that are treated as optimization variables in subproblem $j$, such that $\mathbf{s}_{0}=\left[\mathbf{s}_{1}, \ldots, \mathbf{s}_{M}\right]$ where some $\mathbf{s}_{j}$ may be empty.

\section{Coordination algorithms and convergence properties}

The proposed extensions are special cases of the augmented Lagrangian coordination $\left(\mathrm{ALC}^{8}\right)$ formulation. The nonhierarchical target-response coupling in the ATC formulation are linking variables in the ALC formulation, in which coupling constraints are already included. Hence, the coordination algorithms for ALC proposed in Ref. 8 can be used to solve the decomposed problems and update the penalty parameters $\mathbf{v}$ and $\mathbf{w}$. The theoretical convergence properties of the extended formulation are similar to those for the ALC formulation. Similar to ALC, the convergence proof for the efficient alternating direction method of multipliers, proposed for ATC in Ref. 5, only applies to problem with block-separable system-wide functions. ${ }^{11}$ For non-separable system-wide functions, convergence has only been proven for nested inner loops. 
The non-hierarchical formulation gives the designer freedom to set up the coordination, and to tailor it to an (existing) organizational or computational structure. The price paid for this flexibility is loss of parallelism, since the convergence analysis assumes that subproblems that exchange information are solved sequentially. For purely hierarchical problems, all subproblems at the same level can be solved in parallel since targets and responses are only exchanged between, not within, levels. For non-hierarchical problems, targets and responses may also be exchanged within levels, therefore possibly reducing the degree of parallelization. For coupling functions, additional parallelism is lost due to the coupling of subproblems through the system-wide terms.

\section{Supersonic business jet design example}

A conceptual supersonic business jet design problem serves as a motivating example for the formulation extensions. The example is taken from Ref. 9, and modified versions have been used to demonstrate the use of other coordination algorithms. ${ }^{12,13}$

The optimization problem is concerned with minimizing the weight of the aircraft while considering requirements from the subproblems Structures, Aerodynamics, Propulsion, and Aircraft. Cruise altitude is fixed at $h=55,000 \mathrm{ft}$, and cruise velocity is assumed to be 1.4 Mach. The four subproblems and their data dependencies are displayed in Figure 2. Table 1 gives a brief description of the variables and lists their reference values. These reference values are used to scale the design variables during optimization. The six shared variables $\mathbf{z}$ are design variables in both the aerodynamics subproblem and the structures subproblem, and are depicted in Figure 2 as double arrows. Variables $\mathbf{x}$ are local to one of the subproblem, and the ten coupling variables $\mathbf{y}$ are variables that are computed as outputs of one subproblem that are used as inputs to other subproblems, depicted by directed arrows. The problem has a total of 39 design variables and 46 design constraints. The reader is referred to Ref. 9 for a detailed description of the problem and analysis models. The design optimization problem for the business jet design optimization is given by

$$
\begin{aligned}
\min _{\mathbf{z}, \mathbf{x}, \mathbf{y}} & W_{\mathrm{t}} \\
\text { subject to } & \mathbf{g}_{\text {aircraft }}(\mathbf{y}) \leq \mathbf{0} \\
& \mathbf{g}_{\text {prop }}\left(\mathbf{y}, \mathbf{x}_{2}\right) \leq \mathbf{0} \\
& \mathbf{g}_{\text {aero }}\left(\mathbf{z}, \mathbf{y}, \mathbf{x}_{3}\right) \leq \mathbf{0} \\
& \mathbf{g}_{\text {struc }}\left(\mathbf{z}, \mathbf{y}, \mathbf{x}_{4}\right) \leq \mathbf{0} \\
\text { where } & \mathbf{z}=\left[t / c, A R_{\mathrm{w}}, \Lambda_{\mathrm{w}}, S_{\mathrm{ref}}, S_{\mathrm{ht}}, A R_{\mathrm{ht}}\right] \\
& \mathbf{x}=\left[\mathbf{x}_{1}, \mathbf{x}_{2}, \mathbf{x}_{3}, \mathbf{x}_{4}\right] \\
& \mathbf{x}_{1}=[], \mathbf{x}_{2}=[T], \mathbf{x}_{3}=\left[\Lambda_{\mathrm{ht}}, L_{\mathrm{w}}, L_{\mathrm{ht}}\right], \mathbf{x}_{4}=\left[\mathbf{t}, \mathbf{t}_{\mathrm{s}}, \lambda\right] \\
& \mathbf{y}=\left[L, W_{\mathrm{e}}, W_{\mathrm{t}}, \theta, E S F, D, W_{\mathrm{f}}, L / D, S F C, W_{\mathrm{s}}\right]
\end{aligned}
$$

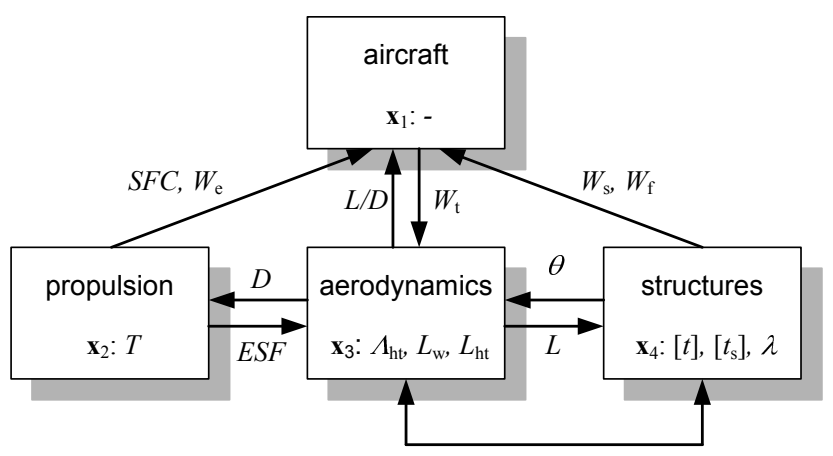

$t / c, A R_{\mathrm{w}}, \Lambda_{\mathrm{w}}, S_{\mathrm{ref}}, S_{\mathrm{ht}}, A R_{\mathrm{ht}}$

Figure 2: Data dependencies for business jet problem. Single arrows indicate direction of response flow, and double arrows indicate shared variables. 
Table 1: Design variables for the supersonic business jet problem. MAC $=$ mean aerodynamic chord, and $W_{s}$ includes all structural weight except engine weight $W_{e}$ and fuel weight $W_{f}$. Reference values refer to the scaling of the variables. Optimal values are listed for the final design obtained with MDF with a weight of $34.3 \cdot 10^{3} \mathrm{lbs}$.

\begin{tabular}{|c|c|c|c|c|c|c|c|}
\hline \multicolumn{2}{|r|}{ shared variables $\mathbf{z}$} & \multicolumn{4}{|c|}{ lower $\leq$ reference $\leq$ upper } & \multirow[t]{2}{*}{ units } & \multirow{2}{*}{$\begin{array}{c}\text { optimal } \\
0.0641\end{array}$} \\
\hline$t / c$ & thickness/chord & 0.01 & $\leq$ & 0.05 & $\leq 0.10$ & & \\
\hline$A R_{\mathrm{W}}$ & wing aspect ratio & 2.5 & $\leq$ & 3.0 & $\leq 8.0$ & \multirow[b]{2}{*}{$\circ$} & 2.5 \\
\hline$\Lambda_{\mathrm{W}}$ & wing sweep angle & 40 & $\leq$ & 60 & $\leq 70$ & & 70 \\
\hline$S_{\text {ref }}$ & wing surf. area & 200 & $\leq$ & 500 & $\leq 800$ & $\mathrm{ft}^{2}$ & 667 \\
\hline$S_{\mathrm{ht}}$ & tail surf. area & 50.0 & $\leq$ & 100.0 & $\leq 148.9$ & \multirow[t]{2}{*}{$\mathrm{ft}^{2}$} & 99.7 \\
\hline$A R_{\mathrm{ht}}$ & tail aspect ratio & 2.5 & $\leq$ & 5.5 & $\leq 8.5$ & & 2.5 \\
\hline \multicolumn{2}{|c|}{ local variables $\mathbf{x}$} & \multicolumn{4}{|c|}{ lower $\leq$ reference $\leq$ upper } & units & optimal \\
\hline$T$ & throttle & 0.1 & $\leq$ & 0.6 & $\leq 1.0$ & \multirow[b]{2}{*}{$\circ$} & 0.196 \\
\hline$\Lambda_{\mathrm{ht}}$ & tail sweep & 40 & $\leq$ & 45 & $\leq 70$ & & 70.0 \\
\hline$L_{\mathrm{W}}$ & wing lift & 0.01 & $\leq$ & 0.15 & $\leq 0.2$ & $\% \mathrm{MAC}$ & 0.01 \\
\hline$L_{\mathrm{ht}}$ & tail lift & 1.0 & $\leq$ & 1.5 & $\leq 3.5$ & $\% \mathrm{MAC}$ & 3.5 \\
\hline $\mathbf{t}$ & 9 thicknesses & 0.1 & $\leq$ & 3.0 & $\leq 4.0$ & inch & 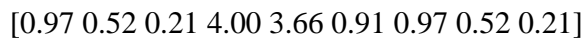 \\
\hline $\mathbf{t}_{\mathrm{s}}$ & 9 thicknesses & 0.1 & $\leq$ & 6.0 & $\leq 9.0$ & inch & 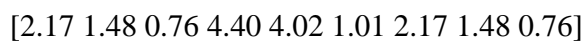 \\
\hline$\lambda$ & taper ratio & 0.1 & $\leq$ & 0.3 & $\leq 0.4$ & & 0.10 \\
\hline \multicolumn{2}{|c|}{ coupling variables $\mathbf{y}$} & \multicolumn{4}{|c|}{ lower $\leq$ reference $\leq$ upper } & units & optimal \\
\hline$L$ & total lift & 5 & $\leq$ & 25 & $\leq 100$ & $10^{3} \mathrm{lbs}$ & 34.3 \\
\hline$W_{\mathrm{e}}$ & engine weight & 0.1 & $\leq$ & 15 & $\leq 30$ & $10^{3} \mathrm{lbs}$ & 6.49 \\
\hline$W_{\mathrm{t}}$ & total weight & 5 & $\leq$ & 25 & $\leq 100$ & $10^{3} \mathrm{lbs}$ & 34.3 \\
\hline$\theta$ & wing twist & 0.2 & $\leq$ & 10.0 & $\leq 50.0$ & $\circ$ & 19.1 \\
\hline$E S F$ & engine scaling factor & 0.5 & $\leq$ & 1.0 & $\leq 1.5$ & & 0.75 \\
\hline$D$ & total drag & 1 & $\leq$ & 40 & $\leq 70$ & $10^{3} \mathrm{lbs}$ & 4.80 \\
\hline$W_{\mathrm{f}}$ & fuel weight & 5 & $\leq$ & 25 & $\leq 100$ & $10^{3} \mathrm{lbs}$ & 10.3 \\
\hline$L / D$ & lift/drag ratio & 0.1 & $\leq$ & 5.0 & $\leq 10.0$ & & 7.03 \\
\hline$S F C$ & spec. fuel cons. & 1.0 & $\leq$ & 2.0 & $\leq 4.0$ & & 1.0 \\
\hline$W_{\mathrm{s}}$ & structural weight & 5 & $\leq$ & 25 & $\leq 100$ & $10^{3} \mathrm{lbs}$ & 17.5 \\
\hline
\end{tabular}

Our implementation of the problem differs from the original version of Ref. 9 to illustrate clearly the proposed formulation extensions. Besides fixing the cruise altitude and cruise velocity, we minimize the total weight instead of the range. A constraint $\mathbf{g}_{\text {aircraft }}$ that requires the range to be at least 2000 nautical miles is added to the problem. Subproblem weights $W_{\mathrm{e}}, W_{\mathrm{f}}$, and $W_{\mathrm{s}}$ are also communicated differently. Here, the structural weight $W_{\mathrm{s}}$ is a newly introduced variable that includes the weight of the total aircraft except the engine weight $W_{\mathrm{e}}$ and fuel weight $W_{\mathrm{f}}$. All three subproblem weights are sent to subproblem Aircraft, which computes the total weight $W_{\mathrm{t}}$ of the aircraft. This weight is then passed to subproblem Aerodynamics.

The optimization problem is solved first using a single-level formulation to set benchmark solutions. We used the multidisciplinary feasible $\left(\mathrm{MDF}^{14}\right)$ approach. The system analyzer in MDF computes the values of $\mathbf{y}$ for fixed $\mathbf{z}$ and $\mathbf{x}$ by performing Gauss-Seidel iterations that run the analyses of Structures, Aerodynamics, Propulsion, and Aircraft sequentially. The problem was solved for 100 different starting points using fmincon (Matlab's SQP solver ${ }^{15}$ ) with default settings and computing gradients by means of the built-in finite difference routine. The objective function $W_{\mathrm{t}}$ is measured in $10^{3} \mathrm{lbs}$, and variables are scaled such that the reference values of Table 1 are equal to 1 . The obtained results indicate that multiple local minima exist. Optimal weight values at each of the three local minima are summarized in Table 2 . The optimal design variable values for the most frequently obtained solution with a weight of $34.3 \cdot 10^{3}$ are listed in Table 1.

\section{A. ATC formulations}

For the analytical target cascading investigations, the problem is first decomposed using a traditional ATC hierarchy in which the top-level subproblem Aircraft coordinates all coupling between its children, each associated with another 
Table 2: Locally optimal weight values obtained with MDF from 100 random starting points within the variable bounds.

\begin{tabular}{|r|c|}
\hline weight $\times 10^{3} \mathrm{lbs}$ & \# of hits \\
\hline 33.0 & 4 \\
34.3 & 83 \\
38.1 & 8 \\
not converged & 5 \\
\hline total & $\mathbf{1 0 0}$ \\
\hline
\end{tabular}

analysis discipline (Propulsion, Aerodynamics, Structures, see Figure 3(a)). Although the decomposition follows the analysis disciplines, the ATC information exchange does not. Any coupling between lower-level subproblems is coordinated at the top level by the introduction of additional target-response pairs. In Figure 3(a) the direction of the single arrows depicts the flow of response variables. The feedback target for $W_{\mathrm{t}}$ coupling Aircraft with Aerodynamics can be included in the ATC formulation of Ref. 6 that allows feedback coupling. Subproblem Aircraft in the ATC decomposition has eighteen variables, whereas its analysis requires only six of them. The other twelve variables are introduced solely for coordination.

By allowing non-hierarchical targets and responses between subproblems, coupling can be coordinated directly between subproblems, and the additional variables do not need to be introduced. A decomposition based on the analysis structure is depicted in Figure 3(b). In this decomposition, each subproblem can send targets to the other disciplines directly, instead of through the top-level subproblem. An additional advantage is that the communication between subproblems follows the analysis dependencies, which may be more natural than the traditional ATC structure. Note, however, that the lower-level subproblems Propulsion and Structures cannot be solved in parallel with subproblem Aerodynamics due to the direct coupling between them. No obvious target-response relation for the shared variables $\mathbf{z}$ is present, since these variables are not responses of either Aerodynamics or Structures. The direction of responses for these variables has no influence on the formulation of each subproblem, and can therefore be chosen arbitrarily.

The use of system-wide constraints can eliminate the specific fuel consumption $S F C$ and the lift-over-drag ratio $L / D$ from the Aircraft subproblem. Since only the range constraint $\mathbf{g}_{\text {range }}$ depends on these variables, coordinating this constraint as a system-wide constraints eliminates them as target variables from the Aircraft subproblem. Instead, $S F C$ becomes a local variable of Propulsion, and $L / D$ is included in the local variables of Aerodynamics. The decomposition with the range as a system-wide inequality constraint is depicted in Figure 3(c), where the box annotated "range constraint" represents the system-wide range constraint $\mathbf{g}_{\text {aircraft }}$. Again, the direction of responses for the shared variables $\mathbf{z}$ is chosen arbitrarily. It is also possible to remove the Aircraft subproblem from the decomposition altogether by including the response relation $W_{\mathrm{t}}=W_{\mathrm{e}}+W_{\mathrm{f}}+W_{\mathrm{s}}$ for subproblem Aircraft as a system-wide equality constraint. The weight variables then become local to each subproblem, as illustrated in Figure 3(d).

To demonstrate the numerical aspects of ATC and the proposed formulation extensions, each of the four decomposed problems is solved from 100 different starting points selected randomly within the variable bounds. For each decomposition, we use an alternating direction ALC algorithm with initial weight selection as presented in Ref. 8. For the initial weight selection, an initial objective estimate of $\hat{f}=10$ is used, together with $\mathbf{w}^{0}=0.001$ and $\alpha=0.1$. The penalty update parameters are set to $\beta=1.05$ and $\gamma=0.95$, and the termination tolerance is $\varepsilon=10^{-3}$. Disciplinary subproblems are solved with fmincon ${ }^{15}$ using default settings. For each run, the obtained optimal weight, the maximum constraint violation, and the required number of subproblem optimizations are taken as performance indicators for the ATC experiments.

The results are listed in Table 3, and show that each decomposition converges to a feasible solution near the local minimum with a weight of $34.3 \times 10^{3} \mathrm{lbs}$. None of the runs converged to the two other local solutions obtained with the MDF experiments. The third and fourth decomposition with system-wide constraints failed to converge to a feasible solution for two starting points.

The number of subproblem optimizations are lower for the second, third, and fourth decomposition when compared to the first (traditional) ATC decomposition, demonstrating that computational cost can be reduced by allowing direct communication between subproblems. This cost reduction can be attributed to the reduction in target-response pairs that have to be coordinated. The first (traditional) ATC decomposition has 26 of these pairs, while the second has only 16 target-response pairs that are coordinated directly between the subproblems. The results show that reducing this number also reduces the required coordination effort. Coordination of the range constraint as a coupling constraint does not show a cost reduction for this example, since it removes only two target-response pairs, and introduces a coupling 


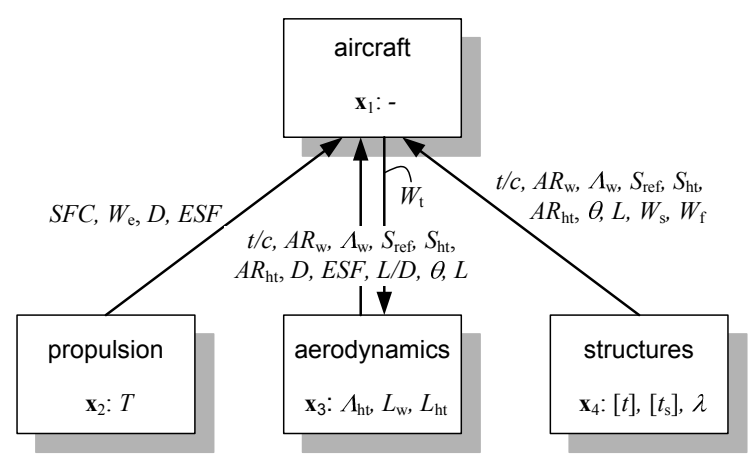

(a) Traditional ATC decomposition with children coupling coordinated by parent. Feedback between Aircraft and Aerodynamics through total weight $W_{\mathrm{t}}$ response of Aircraft.

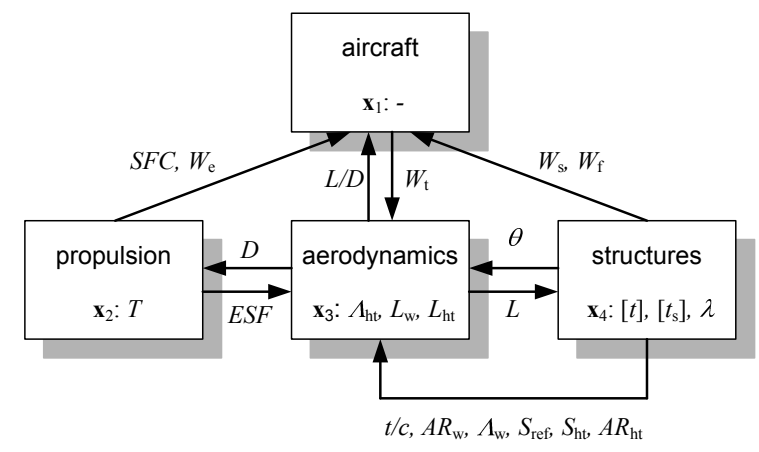

(b) Decomposition with children coupling coordinated directly between children.

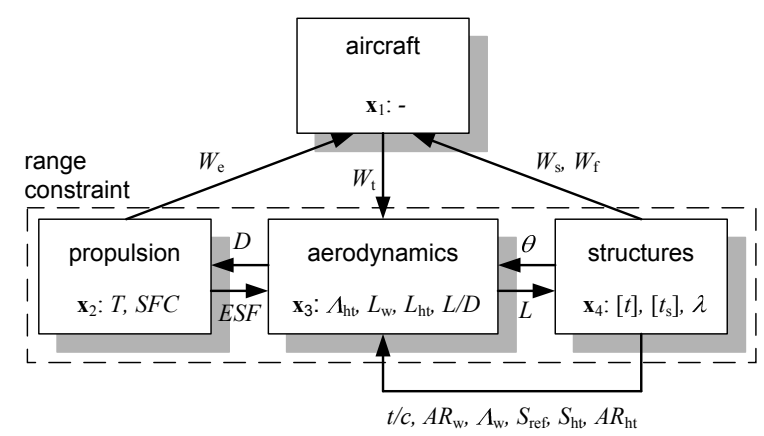

(c) Decomposition with children coupling coordinated directly between children and system-wide range constraint.

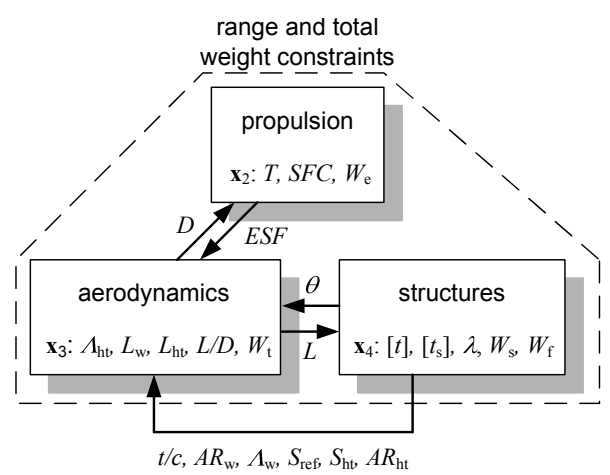

(d) Decomposition with children coupling coordinated directly between children, system-wide range and total weight constraints, and eliminated Aircraft subproblem.

Figure 3: Four problem decompositions. Single arrows represent target-response coupling where the direction of the arrow indicates the direction of response flow. The response directions for the shared variables $t / c, A R_{\mathrm{w}}, \Lambda_{\mathrm{w}}, S_{\text {ref }}$, $S_{\mathrm{ht}}, A R_{\mathrm{ht}}$ for all but the traditional ATC decomposition are chosen arbitrarily. The dashed boxes annotated by "range constraint" in Figure 3(c) and "range and total weight constraints" in Figure 3(d) represent the system-wide inequality constraint $\mathbf{g}_{\text {aircraft }}$ and total weight response equality relation $\mathbf{h}_{\text {aircraft }}=W_{\mathrm{t}}-W_{\mathrm{e}}-W_{\mathrm{f}}-W_{\mathrm{s}}$.

constraint. Eliminating subproblem Aircraft by making the weight response a system-wide equality constraint does reduce computational cost substantially. For this final decomposition, four target-response pairs are eliminated, and only ten pairs have to be coordinated between the three remaining subproblems.

\section{Summarizing remarks}

The two formulation extensions for analytical target cascading (ATC) presented in this paper provide the designer with more flexibility in setting up the coordination structure, while maintaining the advantageous convergence properties of ATC and augmented Lagrangian coordination under standard smoothness and convexity assumptions. The presented example demonstrates that computational benefits can also be gained by selecting inexpensive coordination structures. The flexibility offered by the proposed extensions gives model-based decomposition methods substantial freedom for identifying these inexpensive decomposition structures. Examples of model-based decomposition algorithms are presented in Refs. 16-18. In general, the computational benefits of the extended ATC formulation are expected to be greater for systems with a large amount of non-hierarchical coupling and/or with a few system-wide functions that depend on a large number of local variables. 
Table 3: Optimal weight values, maximum constraint violations, and number of subproblem optimizations obtained for the four decompositions of Figure ?? for 100 random starting points within the variable bounds.

\begin{tabular}{|lr|l|l|l|l|}
\hline & & \multicolumn{4}{|c|}{ Decomposition } \\
\hline & & $(\mathrm{a})$ & $(\mathrm{b})$ & $(\mathrm{c})$ & $(\mathrm{d})$ \\
\hline times converged & & $100 / 100$ & $100 / 100$ & $98 / 100$ & $98 / 100$ \\
\hline \multirow{3}{*}{ optimal weight $\times 10^{3} \mathrm{lbs}$} & $\min$ & 34.3 & 33.9 & 34.1 & 33.9 \\
& $\operatorname{mean}$ & 34.7 & 34.3 & 34.4 & 34.8 \\
& $\max$ & 35.3 & 34.7 & 34.6 & 46.8 \\
\hline \multirow{4}{*}{ max constraint violation } & $\min$ & 0.000 & 0.000 & 0.000 & 0.000 \\
& $\operatorname{mean}$ & 0.002 & 0.002 & 0.001 & 0.002 \\
& $\max$ & 0.005 & 0.008 & 0.006 & 0.004 \\
\multirow{3}{*}{ subproblem optimizations } & $\min$ & 163 & 113 & 111 & 59 \\
& $\operatorname{mean}$ & 187 & 145 & 148 & 71 \\
& $\max$ & 248 & 207 & 204 & 131 \\
\hline
\end{tabular}

\section{A. Acknowledgements}

This work is funded by MicroNed, grant number 10005898. The authors would like to thank Guido Karsemakers for his contribution to the business jet example, and Paul Arendsen and Jeremy Agte for sharing the analysis code for the business jet design problem.

\section{References}

${ }^{1}$ Michelena, N., Kim, H. M., and Papalambros, P. Y., "A System Partitioning and Optimization Approach to Target Cascading," Proceedings of the 12th International Conference on Engineering Design, Munich, Germany, August 24-26 1999.

${ }^{2}$ Kim, H. M., Target Cascading in Optimal System Design, Ph.D. thesis, University of Michigan, 2001.

${ }^{3}$ Michelena, N. F., Park, H., and Papalambros, P. Y., "Convergence Properties of Analytical Target Cascading," AIAA Journal, Vol. 41, No. 5, 2003, pp. 897-905.

${ }^{4}$ Kim, H. M., Michelena, N. F., Papalambros, P. Y., and Jiang, T., "Target Cascading in Optimal System Design,” ASME Journal of Mechanical Design, Vol. 125, No. 3, 2003, pp. 474-480.

${ }^{5}$ Tosserams, S., Etman, L. F. P., Papalambros, P. Y., and Rooda, J. E., "An Augmented Lagrangian Relaxation for Analytical Target Cascading Using the Alternating Direction Method of Multipliers," Structural and Multidisciplinary Optimization, Vol. 31, No. 3, 2006, pp. $176-189$.

${ }^{6}$ Allison, J. T., Kokkolaras, M., Zawislak, M. R., and Papalambros, P. Y., "On the Use of Analytical Target Cascading and Collaborative Optimization for Complex System Design," Proceedings of the 6th World Congress on Structural and Multidisciplinary Optimization, Rio de Janeiro, Brazil, 30 May - 3 June 2005.

${ }^{7}$ Kim, H. M., Chen, W., and Wiecek, M. M., "Lagrangian Coordination for Enhancing the Convergence of Analytical Target Cascading," AIAA Journal, Vol. 44, No. 10, 2006, pp. 2197-2207.

${ }^{8}$ Tosserams, S., Etman, L. F. P., and Rooda, J. E., “Augmented Lagrangian Coordination for Distributed Optimal Design in MDO,” International Journal for Numerical Methods in Engineering, Vol. 73, No. 13, 2008, pp. 1885-1910.

${ }^{9}$ Agte, J. S., Sobieszczanski-Sobieski, J., and Sandusky, R. R. J., "Supersonic Business Jet Design through Bi-level Integrated System Synthesis," Proceedings of the World Aviation Conference, San Fransisco, CA, 1999, MCB Press, SAE paper 1999-01-5622.

${ }^{10}$ Kokkolaras, M., Fellini, R., Kim, H. M., Michelena, N. F., and Papalambros, P. Y., "Extension of the Target Cascading Formulation to the Design of Product Families," Structural and Multidisciplinary Optimization, Vol. 24, No. 4, 2002, pp. 293-301.

${ }^{11}$ Tosserams, S., Etman, L. F. P., and Rooda, J. E., "Block-separable Linking Constraints in Augmented Lagrangian Coordination," Structural and Multidisciplinary Optimization, 2008, Published online. DOI: 10.1007/s00158-008-0244-5.

${ }^{12}$ Sobieszczanski-Sobieski, J., Altus, T. D., Phillips, M., and Sandusky Jr., R. R., "Bilevel Integrated System Synthesis for Concurrent and Distributed Processing," AIAA Journal, Vol. 41, No. 10, 2003, pp. 1996-2003.

${ }^{13}$ Tosserams, S., Etman, L. F. P., and Rooda, J. E., "Numerical performance Evaluation for Augmented Lagrangian Coordination for Distributed Multidisciplinary Design Optimization," Proceedings of the 4th AIAA Multidisciplinary Design Optimization Specialist Conference, Schaumburg, IL, 7-10 April 2008, AIAA paper 2008-1805.

${ }^{14}$ Cramer, E. J., Dennis, J. E., Frank, P. D., Lewis, R. M., and Shubin, G. R., "Problem Formulation for Multidisciplinary Optimization,” SIAM Journal on Optimization, Vol. 4, No. 4, 1994, pp. 754-776.

${ }^{15}$ Mathworks, Matlab User Guide, Version 7, Mathworks Inc., Natick, MA, 2008.

${ }^{16}$ Michelena, N. F. and Papalambros, P. Y., "A Hypergraph Framework for Optimal Model-based Decomposition of Design Problems," Computational Optimization and Applications, Vol. 8, No. 2, 1997, pp. 173-196.

${ }^{17} \mathrm{Li}$, S. and Chen, L., "Model-Based Decomposition Using Non-Binary Dependency Analysis and Heuristic Partitioning Analysis," Proceedings of the ASME Design Engineering Technical Conferences, Philadelphia, PY, September 2006. 
${ }^{18}$ Allison, J. T., Kokkolaras, M., and Papalambros, P. Y., "Optimal Partitioning and Coordination Decisions in System Design Using an Evolutionary Algorithm," Proceedings of the 7th World Congress on Structural and Multidisciplinary Optimization, Seoul, South Korea, 21 - 25 May 2007. 